Une troisième version du "Dicautom ".

J.A. Bachrach et L. Hirschberg.

Notre dictionnaire automatique fournit la traduction des termes d'un texte, commentée par des exemples, à des équipes de traducteurs et d'interprètes qui travaillent sur les quatre langues de la Communauté Européenne et sur 1'anglais.

Il se différencie d'un dictionnaire, fait de papier et de carton, essentiellement par les moyens automatiques dont il dispose pour le tri, le stockage, la mise a jour, la consultation ou l'impression. Mais s'il est plus riche en exemples et manipulé en ordinateur, il a été réuni par des lexicologues, des terminologues et des traducteurs, comme un dictionnaire ordinaire.

L'équipe responsable des versions successives se composait des membres du Bureau de Terminologie de la Haute Autorité de la Ceca, J.A. Bachrach, R. Denti, P. Fleurbaay, J. Goetschaelckx, H. Lenoch, C. Noss, R. Weisgerber, J. Wilson, et du Centre de Linguistique Automatique Appliquée de l'Université libre de Bruxelles, J. Blois, Fr. Decresy, L. Delhaye, R. Dorrepaal-Van Beek, L. Hirschberg, N. Hollaar, J. Mommens, E. Morlet.

La description présentée ici concerne une troisième version du système, fonctionnant sur une IBM 360/40, achevée en décembre 1966 et mise au point au départ de la langue allemande. La première version sur IBM 1401 et au départ du français, date de 1962 (" La Traduction Automatique " - IV-3-1963). La deuxième a été présentée a Venise par J.A. Bachrach au " Séminaire International de l'OTAN sur la Documentation Automatique " (juillet 1963) et publiée depuis en divers endroits. Elle fonctionne sur IBM 1410, au départ du français et du néerlandais. Ainsi qu'il ressort du rapport " Language and Machine ", publié fin 1966 par la National Academy of Sciences, Washington, il n'existe qu'une seule formule qui se rapproche de la nôtre, à savoir celle adoptée par le service de traduction de l'armée allemande à Mannheim.

Au cours de cette nouvelle expérience nous avons précisé les définitions des concepts linguistiques, simplifié et systématisé les codages manuels. Nous avons réanalysé et transformé le processus et réorganisé les fichiers. Enfin, en tenant compte des possibilités nouvelles de la machine, d'une mémoire de $256 \mathrm{~K}$, et en fixant les modes d'exploitation des programmes, nous en avons considerablement amélioré le rendement.

Avant d'entrer dans le détail de la description du système, il est bon de donner un aperçu de son utilisation et des motivations qui ont guidé sa conception. 
Notre dictionnaire comporte un mot ou une locution de la langue source, sa ou ses traducti ons dans les langues cibles, et pour chaque unité des exemples de contextes et leurs traductions. Ce qui est valable pour la langue générale, l'est également pour la langue technique; la traduction d'un terme ne dépend pas seulement du domaine, de la matière traitée, de l'époque ou il a été employé, mais aussi des contextes dans lesquels' il se place. Toutes ces données sont réunies dans le dictionnaire. Quelle sera la source de nos exemples? Les terminologues puisent de préférence leurs informations dans des documents originaux: manuels, rapports, catalogues, brochures scientifiques ou publicitaires et surtout dans les revues specialisées. L'emploi de textes traduits en plusieurs langues serait plus simple, mais risquerait d'entrainer la confection d'un langage artificiel et de perpetuer des erreurs. Il importe de se servir dans chaque langue de la terminologie propre au secteur en question dans le pays d'origine de cette langue.

Le terminologue reçoit du traducteur des questions, sous forme de phrases-demandes ou de fragments de phrases dans lesquelles le traducteur a senti des pieges de traduction. Du stock de phrasesexemples que nous avons accumulees dans notre fichier - qui s'elève à quelque six mille unités aujourd'hui, mais peut arriver à cent mille - l'ordinateur doit extraire les exemples de traduction les "plus proches possibles" de ceux de la demande. La présence des contextes dans les réponses permet au traducteur de choisir lui-même la traduction des mots, en contrôlant le bien-fondé des traductions proposées par les exemples, relativement aux cas qui l'occupent. S'il y a doute, plusieurs réponses sortiront, parmi lesquelles le traducteur éliminera lui-même les réponses inadéquates. (Illustration I)

Une autre condition importante pour l'utilisateur est que les questions puissent être formulees librement. En aucun cas, la formulation de la question ne nécessite une connaissance mềne partielle de la réponses: tout 1 'effort de construction du système incombe au réalisateur. L'utilisateur souligne simplement un fragment de quelques mots. Pour que ce fragment de texte à traduire puisse être découpé sans précautions particulières, les terminologues doivent veiller à opérer un découpage judicieux pour obtenir les phrases-exemples.

L'absence de tout codage au moment de la demande, implique un codage effectué lors de la construction du dictionnaire, qui donne des lois d'identification des formes du texte courant avec les unités du lexique. Une telle structure se justifie par le fait que le programme est conçu pour consulter, frequemment et en vue d'une très grande quantité de textes à traduire, un dictionnaire de phrases relativement limite, de sorte que le codage a l'entrée est le plus économique. (Illustration II) 


\section{Illustration I}

\section{demande 0000000012}

eine $\$$ traenke dieser art besteht aus einem robusten verzinkten $\$$ stahlblechgehaeuse

\section{réponses}

traenke

als automatische traenken haben sich tropftraenken und rinnentraenken bewaehrt

fra gli abbeveratoi automatici si sono affermati quelli a goccia e quelli a livello costante

bestehen aus blech

die verteilerkoepfe der melkzeuge bestehen aus gedrueckten nichtrostenden stahiblechteilen

i distributori delle unita di mungitura sono compos$E_{i}$ di pezzi a rilievo in lamiera di acciaio inossidabile

bestehen aus luftheizungsoefen bestehen aus einem inneren ofen$\operatorname{stahl}$ blech mantel aus stahl und einem aeusseren stahlblechmantel

le stufe ad aria calda sono costituite di un rivestimento del forno in acciaio e di un rivestimento metallico esterno

stahl selbsttragende pfannen aus verzinktem stahlblech

blech

verzinken elementi autoportanti in lamiera d acciaio zincata

blech ventilatoren fuer unterdachtrocknung werden zum gehaeuse ortsfesten einbau in stahlblechgehaeuse montiert geliefert

$i$ ventilatori per 1 essiccamento dell erba in fienile sono disponibili con involucri in lamiera d accidemande 0000000013 aio per montaggio stabile nel fienile

hochfoerderrinne mit schubstangen und $\$$ sperrechen

pas de réponse. 
Trois modes de consultation paraissent adequats selon le rythme et le volume des questions.

" En direct " tout d'abord, le programme se trouve en permanence tout entier dans la machine, munie de tous les fichiers, et simultanément d'ailleurs avec des programmes appeles pour d'autres travaux. Le demandeur possede sur sa table un clavier d'appel qu'il actionne a tout moment et meme pour une seule question. La réponse apparalt sur un écran de telévision ou se dactylographie sur une machine a ecrire. Nous arriverons a ce mode de fonctionnement lorsque notre ordinateur sera organise en multiprogranmation et lorsque nous posséderons des unités de stockage permanent pour nos dictionnaires. $I$ 'organisation actuelle permet de travailler " en differe ". c'est-à-dire de réunir jusqu'à mille phrases demandées en une journee, les faire perforer par une dactylo sur des cartes IBM et de distribuer les réponses quelques heures plus tard.

Mais une organisation bien plus efficace, lorsqu'il s'agit de traduire systématiquement de grands volumes de textes, pourra Etre mise en oeuvre bientót. Il existe deja en Europe des imprimeries qui font corriger et justifier automatiquement en ordinateur les textes a'imprimer. Au moment ou 1 'imprimeur passe les textes de la bande linotype en ordinateur nous pouvons les introduire et les conserver sur bandes magnétiques sans avoir à les perforer. Dès lors si ces textes sont destinés à être traduits, nous pouvons les passer sans aucun travail humain supplémentaire sur notre programme de consultation de dictionnaire. A la sortie, nous obtiendrons, en plus et indépendampent du texte imprime, une préparation du volume entier, qui nous informera, phrase par phrase. sur les difficultés de traduction vers une ou plusieurs langues et la maniere de les surmonter.

Mais avant que nous ne soyons en possession de cet immense fichier et d'une réponse instantané a toutes nos questions sur un écran de télevision les terminologues doivent répondre aux besoins journaliers par des moyens plus classiques. En aucun cas leurs obligations courantes ne peuvent etre diminuees ni ralenties sous pretexte de construction pour le futur. Par ailleurs une realisation à longue echeance ne peut être effectué serieusement en marge de la connaissance des besoins pratiques actuels. C'est pourquoi nous nous servons de leur propre fichier, pour notre dictionnaire automatique, mais aussi pour 1 'edition de glossaires phraseologiques a l'aide d'un programme qui evite toutes les manipulations ordinaires de la typographie.

Ce que le terminologue introduit dans la machine, pour construire le dictionnaire c'est le contenu d'une fiche, c'est-àdire un fragment de texte plus ou moins long (jusqu'a vingt mots environ) ainsi qu'un code-matiere et une reférence bibliographique. 


\section{Illustration II}

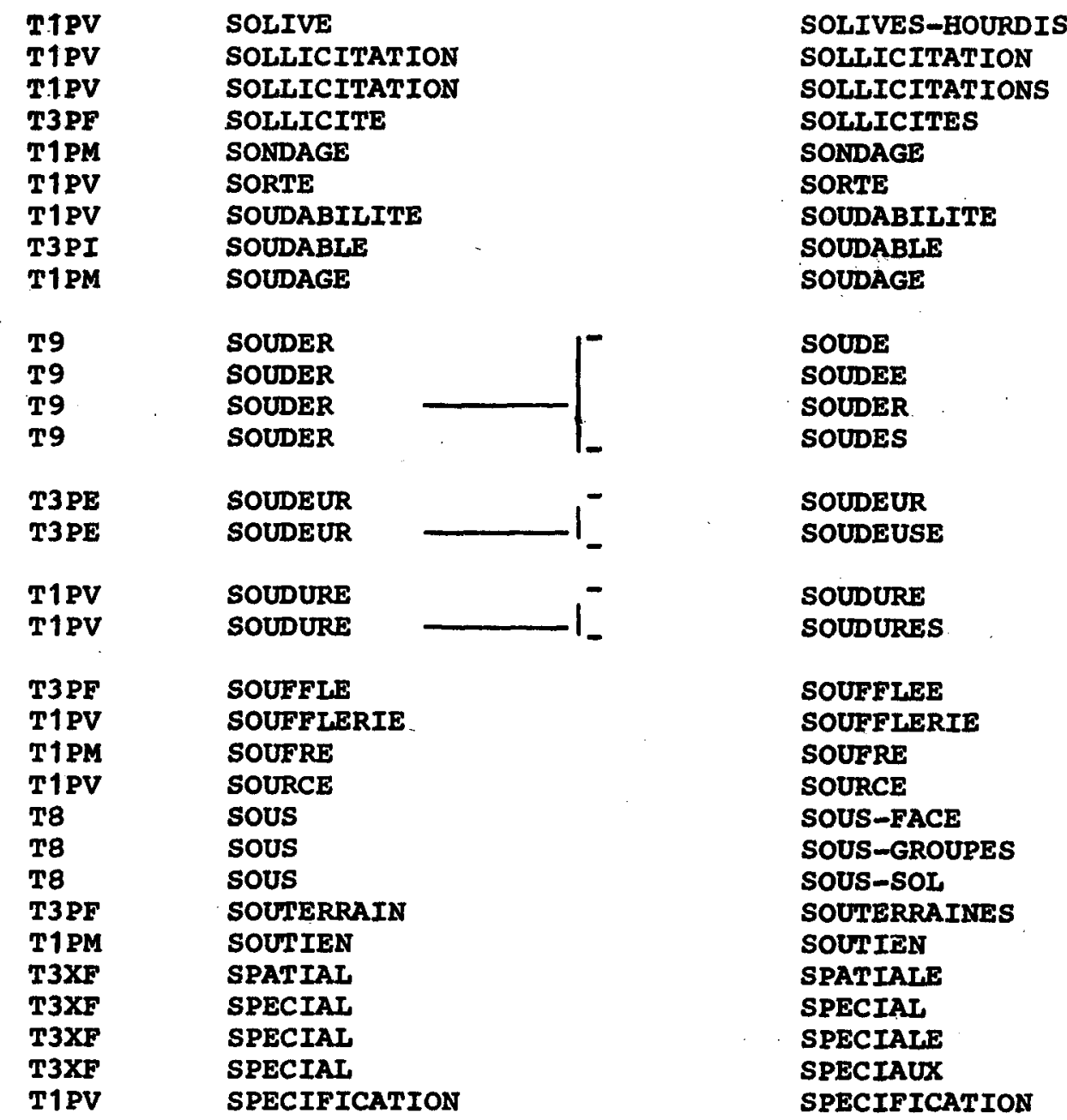


Ce fragment contient une difficulté de traduction et le contexte qui conditionne l'emploi de cette tournure particuliere. Si nous avons en français, " PONT A POUTRES EN TREILLIS " , le même Enoncé est repris dans cing ou six langues sur la même fiche. Il servira de"phrase-exemple" (au singulier) qui ira dans le fichier.

\section{F PONT A POUTRES EN TREILLIS}

D FACHWERKBALKENBRUECKE

I PONTE A TRAVI A TRALICCIO

(Illustration III)

N VAKWERKLIGGERBRUG

E TRUSSED GIRDER BRIDGE

Le premier travail de la machine est effectue par un programme du genre "KWIC" qui attache cette fiche toute entiere, avec les énoncés, dans toutes les langues, en autant d'endroits différents qu' elle contient de mots: pont, poutres, treillis, vakwerkliggerbrug, bridge etc. En chaque endroit, la fiche va rejoindre d'autres fiches conçues comme elle. (Illustration IV)

En somme, le même stock de phrases-exemples, existant actuellement en cing langues, est utilise pour cing dictionnaires, un dictionnaire par langue de depart. Toutefois, le programme est prévu pour six langues. Il s'ensuit que cinq réponses peuvent etre donnés à partir d'une langue, vers cing langues differentes. Cela equivaut finalement a trente dictionnaires bilingues.

Insistons maintenant sur le fait que les listes-dictionnaires finales contiennent des "unites lexicales" et non pas des mots, car ces unités sont parfois plus longues, comme "plaque de façade" ou "sauerstoffgeblasenes Eisen". tenus pour des unités. Parfois elles'sont plus courtes. Ainsi il faut trois unites lexicales "Rost" "Stab" et "Eisen" pour reconstituer automatiquement c'est-à-dire reconnartre le mot "Roststabeisen" dans le texte $d$ 'une demande.

c'est le constructeur qui choisit 1 'unité lexicale et marque pour chacune d'elles les phrases du stock qui la concernent. Il emploie des critères d'observation basés sur les phrases-exemples et leurs traductions.

Lorsqu'un mot se traduit de plusieurs manières différentes, notre système donne au lexicologue le choix entre deux solutions. Ou bien il crée pour le mot plusieurs rubriques, chaque rubrique contenant d'autres phrases-exemples, et nous disons qu'il s'agit d'autant d" "homonymes" différents de ce mot. Ainsi en allemand "Raum" se traduira dans de nombreux contextes par "espace". dans d'autres par "chambre" ou "local" et enfin nous lui avons même trouve la traduction "surface". Nous créons des "homonymes". lorsque le nombre de traductions $n$ 'est pas trop grand, et les . 6 . 
contextes ou ces traductions s'imposent sont nombreux.

Au contraire, nous avons trouve pour "Lager" plusieurs traductions très particulières qui ne convenaient chacune qu'à un contexte bien défini. Pour définir les cas ou il fallait choisir ces traductions, le mieux était de faire des "groupes locutionnels" de ces contextes, c'est-à-dire des unités indépendantes composées de plusieurs autres unités. Notre lexique allemand contient de cette manière "Kugellager" (roulement à bille), "Gleitlager" (appui glissant). "Obstlagerhaus" (dépôt ou entrepôt de fruits). "Lagerbehaelter" (cellule de stockage).

\section{Illustration IV}

PONT

PARAPETS DE ROUTES ET DE PONTS

D STRASSEN - UND BRUECKENGEIAENDER

I PARAPETTI STRADALI E DI PONTI

N BORSTWERINGEN VOOR WEGEN EN BRUGGEN

E ROAD AND BRIDGE RAILINGS

PONT

PONT A POUTRES EN TREILLIS ET ARTICULATIONS INTERMEDIAIRES

D FACHWERKBALKENBRUECKE MIT ZWISCHENGELENKEN

I PONTE A TRAVI A TRALICCIO ARTICOLAZIONI INTERMEDIE

N VAKWERKLIGGERBRUGGEN MET SCHARNIERENDE DIAGONALEN

E LATTICE GIRDER BRIDGES, WITH INTERMEDIATE JOINTS

PONT

PONT A POUTRES EN TREILLIS

D FACHWERKBALKENBRUECKE

I PONTE A TRAVI A TRALICCIO

N VAKWERKLIGGERBRUG

E TRUSSED GIRDER BRIDGE

PONT

PONT BASCULANT

D KLAPPBRUECKE

I PONTE LEVATOIO., PONTE RIBALTABILE

N KLAPBRUG. , OPHAALBRUG. , BASCULEBRUG

E BASCULE BRIDGE

PONT

PONT CANTILEVER EN TREILLIS

D FACHWERK-GERBERTRAEGER-BRUECKE

I PONTE A SBALZO A TRALICCIO

N VAKWERK-GERBERLIGGERBRUG

E CANTILEVER TRUSS BRIDGE 
Les "groupes locutionnels", comme les autres unités lexicales, possèdent dans notre dictionnaire des phrases-exemples qui leur sont attachées, Mais il s'agit de reconnattre de telles unités dans le texte d'une demande.

C'est donc par une analyse morphologique au sens le plus large de ce terme, allant de la reconnaissance d'une forme et de sa découpe à la découverte d'un groupement de plusieurs formes dans une phrase, que commencent les programmes de consultation automatique de nos dictionnaires. Ils comportent deux phases importantes en dehors de routines de tri et de mises en page, la deuxième grande étape étant le choix des meilleures phrases-exemples à donner en réponse à une certaine demande. Ce choix est fait parmi les phrases attachées aux rubriques du dictionnaire qui viennent d'être reconnues par l'analyse morphologique.

Les phrases-réponses les meilleures sont choisies par programme. Nous avons élaboré dans ce but des règles précises et un calcul assez compliqué. Mais en un mot il est permis de dire que ce sont les phrases parmi celles du stock qui ont en elles chacune le plus grand nombre possible d'unités lexicales reconnues dans une demande par la machine.

Le dictionnaire se compose de quatre fichiers indépendants, ce qui accélère la consultation automatique et facilite la correction éventuelle de ces fichiers.

Le premier est un dictionnaire alphabétique de mots ou de radicaux, accompagnés de leurs codes morphologiques et de deux numéros de renvoi, le numéro de la liste des groupes locutionnels auxquels ils appartiennent et le numéro de la liste des phrases-exemples dans lesquelles ils apparaissent comme mots simples. $L$ 'une des deux listes peut d'ailleurs ne pas exister.

Ce premier fichier est suffisamment dépouillé pour qu'il puisse être chargé en mémoire rapide au moment de la consultation du dictionnaire. Une table d'adresses constituée par les deux premières lettres des mots se forme au mềne moment dans cette mémoire. Cela permet une analyse morphologique très rapide, sans même trier alphabétiquement les mots d'une demande. (Illustration $v$ )

Toutes les hypothèses de découpes sont faites pour chaque mot, de gauche a droite. Le programme tient compte aussi de l'existence possible, de désinences finales à la fin d'une séquence de lettres.

De plus il suit des règles d'acception et de rejet des diverses découpes, dont nous ne donnerons qu'un exemple d'application. Ainsi on voit dans l'illustration $v$, que " SERIEN|BAUTEN " a été découpée en deux mots, tandis que la découpe "BAU|KASTEN/SYSTEM": a été rejetée (un signe "moins" se trouve devant le sigle DE) au profit de la découpe "BAUKASTEN/SYSTEM". Il est évident qu'il refuse aussi toute hypothèse qui ne recouvre pas convenablement une séquence de lettres sans blancs. 


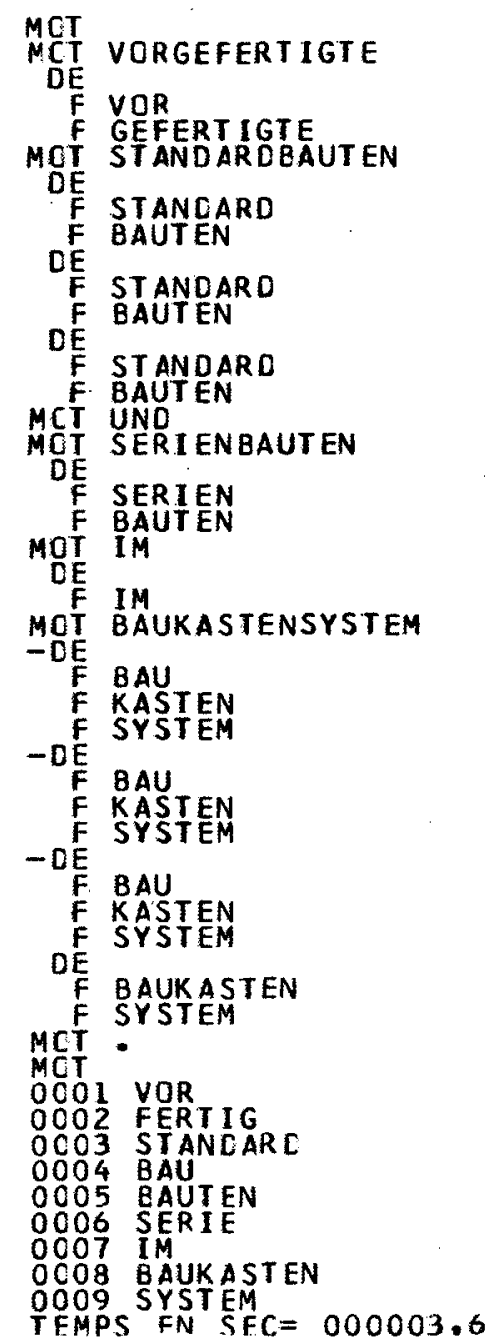

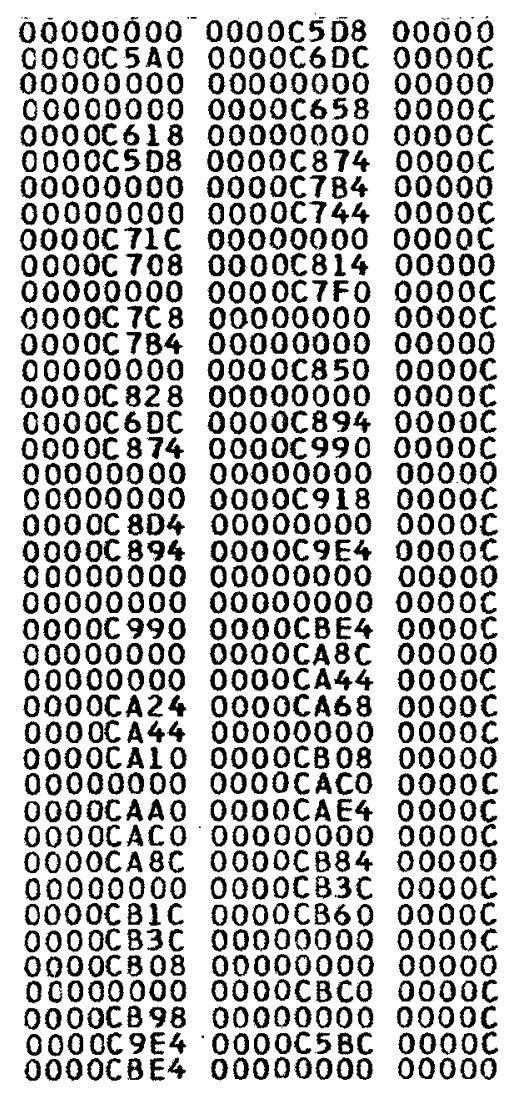


Tout le processus n'a duré que 3,6 secondes pour une demande de six mots dont quatre ont été découpés en deux.

Le numéro de liste des groupes locutionnels est le premier argument de tri du deuxième fichier, qui donne ainsi pour chaque mot les numéros des groupes locutionnels auxquels il appartient dans le dictionnaire. Les mots eux-mêmes ne réapparaissent pas dans cette deuxième liste, mais seulement leurs caractéristiques relativement à chaque groupe, qui permettent de vérifier si les conditions d'existence du groupe sont remplies dans un texte que l'on analyse. (Illustration VI)

Après la consultation des deux premiers fichiers, les mots d'une demande ont été découpés en fragments ou (et) réunis en groupes locutionnels. Chaque fragment peut avoir aussi été identifié avec plusieurs homonymes.

A chaque homonyme libre, comme à chaque fragment d'un groupe locutionnel, correspond une liste des numéros des phrases-réponses valables pour lui. C'est le troisième fichier qui contient ces listes de numéros de phrases, sans contenir les phrases elles-mêmes. Il mentionne aussi les langues-cibles pour lesquelles ces phrases existent. On remarquera le gain de temps obtenu en effectuant l'analyse jusqu'ici sans se soucier de la langue cible. Ce n'est qu'au moment de la consultation du troisième fichier pour la recherche de la meilleure phrase-réponse que se fait la séparation du processus par langues demandées.

Après consultation de ce troisième fichier, le programme attache à chaque radical découpé dans une demande plusieurs paquets de numéros de phrases-réponses, chaque paquet correspondant à un homonyme ou à une hypothèse de groupe locutionnel. On obtient ainsi un tableau de numeros de phrases ou chaque phrase est citée un certain nombre de fois. Nous attribuons à chaque phrase une cote le plurs souvent égale à ce nombre, moyennant certaines précautions dans les cas de découpes multiples.

On choisit ensuite pour chaque fragment de la demande la phraseréponse qui le concerne et qui possède la cote la plus élevée. Cela,constitue en meme temps un choix parmi ses homonymes. Cependant plusieurs homonymes peuvent être acceptés, avec la meilleure phrase-: réponse de chacun d'eux, si elles ont toutes la même cote.

$\mathrm{L}$ 'appartenance $a$ un groupe locutionnel n'élimine pour un mot toutes ses autres hypothèses que si ce groupe $a$, dans le texte envisagé, une valeur de certitude qui est determinée par programme. On se base sur des critères de certitude que nous ne pouvons énumérer ici. Disons par exemple qu' un verbe séparable n'est reconnu avec certitude que dans le cas ou la particule est collée au radical verbal. Dans les autres cas on permet à plusieurs hypothèses d'entrer en competition.

Ce $n$ 'est qu'au moment d'éditer les résultats que $1^{\prime}$ on retire du dernier fichier les phrases-exemples elles-mêmes, qui viennent d'etre choisies. 


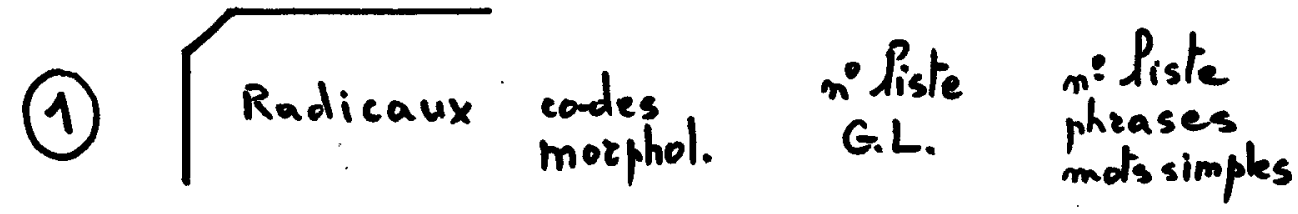
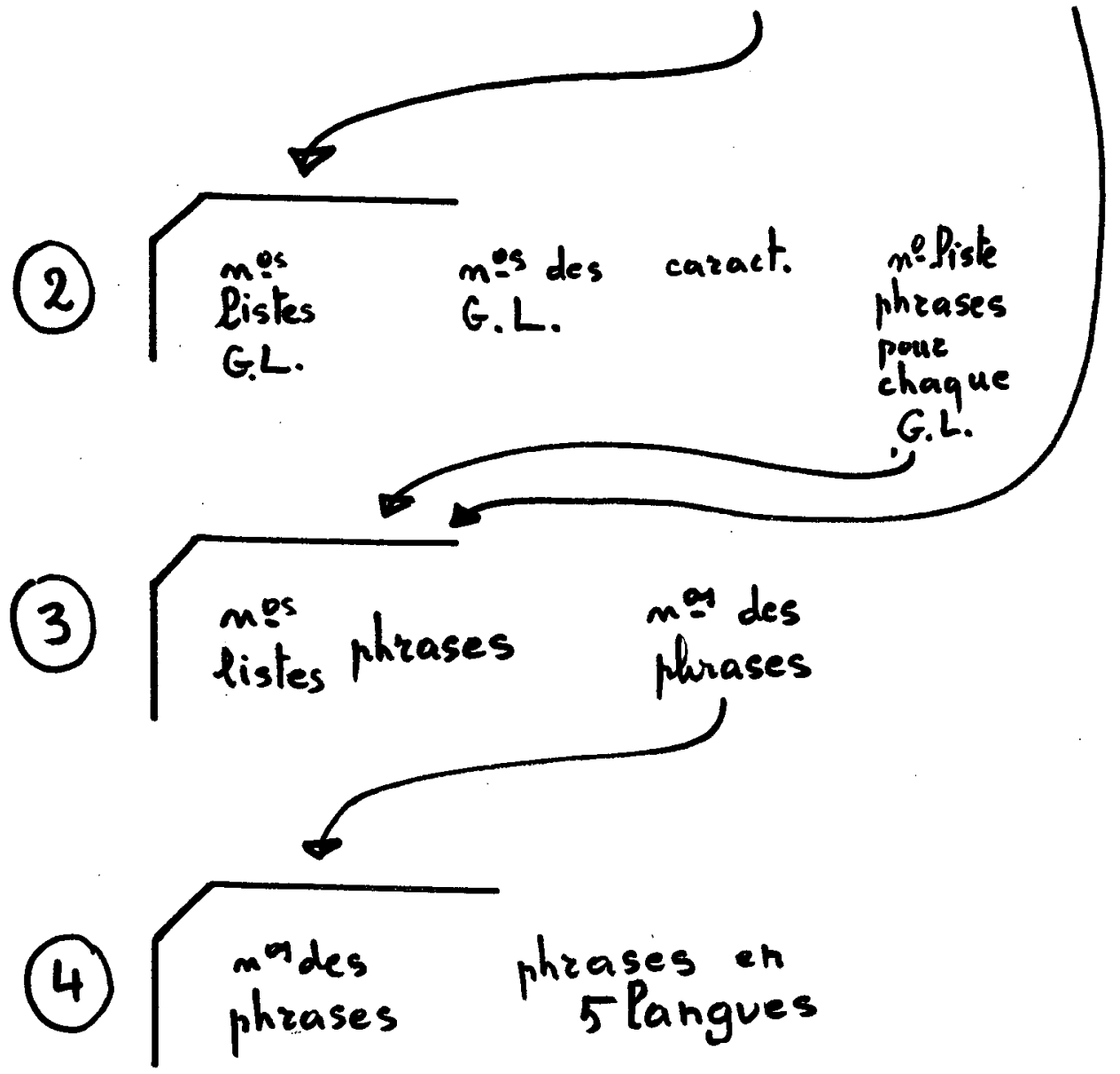
Ce quatrième fichier, trié par numéros de phrases, peut être beaucoup plus volumineux que les autres et tenir, s'il le faut, dans une mémoire-mars.

Il nous reste à justifier quelques détails de l'analyse morphologique adoptée ici. Elle se caractérise tout d'abord par la comparaison des unités lexicales à partir de la gauche.

Les avantages de reconnaissance des formes à partir de la gauche sont liés au fait que la machine consulte d'abord le lexique des formes, et non pas des listes fermées de désinences, en commençant par la droite. Le premier avantage, sauf pour des unités lexicales de moins de quatre lettres et des préfixes, réside dans le fait que la machine va plus vite, parce qu'elle crée beaucoup moins d'hypothèses inutiles, ainsi que nous 1 'avons vérifié expérimentalement, en simulant les deux méthodes.

Mais il y a un deuxième avantage de loin le plus important surtout pour des langues comme I'allemand et le neerlandais qui agglomèrent en un mot des unités lexicales que l'on peut, -et que l'on doit, séparer dans le lexique. En effet le programme peut effectuer en une seule fois les découpes des mots et des désinences, jusqu'à épuisement et sans retour en arrière.

Enfin, nous avons groupé les innombrables déclinaisons des substantifs allemands en admetant une seule liste de désinences pour tous, définie par le code signifiant substantif. (Illustration VII)

La simplification des déclinaisons allemandes au point de les ramener toutes à une seule, facilite énormément le codage du dictionnaire, si nous le comparons au codage nécessaire pour 1'analyse morphologi-: que élaborée par le groupe de Grenoble, qui tient compte de vingttrois listes distinctes de désinences pour les substantifs allemands.

Que sacrifions-nous à cette simplification?

Il n'y a aucun danger, dans le cas présent, à admettre pour un substantif des désinences qu'il ne prendra en réalité jamais. En effet si cela pouvait mener à confondre deux substantifs, il suffirait de donner toutes leurs formes dans le dictionnaire et de les traiter comme des mots invariables. Nous $n$ 'avons pas rencontré cette situation sur plusieurs milliers de mots de textes techniques traités ainsi. De toutes manières, dans le but d'obtenir une découpe rapide des composés, faite de gauche à droite et en un seul passage, nous donnons deux formes dans le dictionnaire pour les substantifs qui prennent un "umlaut" ou pour tout autre changement de radical.

Notre analyse perd une seule information, celle de savoir à quel cas et à quel nombre appartient un substantif donné dans un texte. Elle convient donc pour les applications pour lesquelles ces renseignements seraient superflus, telles que les consultations automatiques de dictionnaires qui ne seraient pas suivies d'analyses syntaxiques. 


\section{Illustration VII}

\begin{tabular}{|c|c|c|c|c|c|c|c|c|}
\hline $\begin{array}{c}\text { mots qui } \\
\text { les acceptent } \\
\therefore\end{array}$ & \multicolumn{2}{|c|}{ noms } & \multicolumn{2}{|c|}{ adjectifs } & forts & \multicolumn{2}{|c|}{$\begin{array}{l}\text { verbes } \\
\text { faibles /participes }\end{array}$} & \\
\hline désinences & F & I & FetI & $\mathrm{F}$ & $F$ & $F$ & $\mathrm{~F}$ & $I$ \\
\hline $\begin{array}{l}\text { blanc } \\
\text { CHEN } \\
\text { CHENS } \\
\text { E } \\
\text { EM } \\
\text { EN } \\
\text { END } \\
\text { ER } \\
\text { ES } \\
\text { EST } \\
\text { ET } \\
\text { ETE } \\
\text { ETEN } \\
\text { ETEST } \\
\text { ETET } \\
\text { LEIN } \\
\text { LEINS } \\
\text { N } \\
\text { ND } \\
\text { NEN } \\
\text { R } \\
\text { S } \\
\text { SE } \\
\text { SEN } \\
\text { SES } \\
\text { ST } \\
\text { T } \\
\text { TE } \\
\text { TEN } \\
\text { TEST } \\
\text { TET }\end{array}$ & $\mid \begin{array}{l}0 \\
0 \\
0 \\
0 \\
0 \\
0 \\
0 \\
0\end{array}$ & $\begin{array}{l}0 \\
0 \\
0\end{array}$ & 0 & $\begin{array}{l}0 \\
0 \\
0 \\
0 \\
0\end{array}$ & 0 & $\begin{array}{l}0 \\
0 \\
0 \\
0 \\
0 \\
0 \\
0 \\
0 \\
0 \\
0 \\
0\end{array}$ & $\begin{array}{l} \\
0 \\
\vdots \\
\vdots \\
0\end{array}$ & ० \\
\hline
\end{tabular}

$F=$ finales $\quad I$ = intermédiaires 
Au moment de conclure, il est bon de passer en revue quelques points que soulève la lexicographie classique et de voir dans quelle mesure ils nous concernent ici.

Il s'agit principalement du choix des termes à introduire dans un dictionnaire, c'est-à-đire de la délimitation d'un domaine sémantique et du relevé du plus grand nombre possible de termes que s'y rapportent, ensuite des relations sémantiques entre les termes c'est-àdire de la structuration sémantique à l'intérieur du lexique choisi; et enfin des problèmes d'adressage, c'est-à-dire de l'organisation matérielle en vue de la consultation, emplacement des termes, répétition éventuelle, orthographes multiples etc...

Si nous n'avons pas centré notre exposé sur ces questions, c'est que le traitement en ordinateur les résoud assez naturellement à condition d'introduire en machine les données complètes et primitives.

L'adressage tout d'abord est à ce point aisé que 1 'on peut multiplier a souhait les orthographes et que les problèmes d'emplacement et de répétition ne se posent plus.

En introduisant en machine des fragments de textes authentiques, nous notons en même temps leur référence bibliographique et un ou plusieurs codes matières définissant le contexte dont ils ont été extraits. Ces informations sont par là-mème et sans autre travail attachées aux termes contenus dans ces fragments. Comme la mémoire de la machine est pratiquement illimitée, on ne doit refuser aucun terme a l'entrée, ni séparer explicitement des domaines, tels la sidérurgie, l'architecture etc... Tout au plus devra-t-on attacher à certaines unités lexicales un signe spécifiant qu'elles n'ont aucun intérêt par elles-mêmes dans un dictionnaire technique (par exemple pour des mots comme "faire", "grand", "froid" etc.).

Une liste parfois longue de codes-matières se trouve attachée à chaque unité par le truchement des diverses phrases-exemples. Il s'etablit ainsi spontanément une structuration sémantique complexe à l'intérieur du système que l'on pourrait découvrir par des techniques taxonomiques. Elle est plus riche que la structure en arborescence (analogue à la classification décimale universelle)la seule qui soit réalisable dans un dictionnairé habituel, a côté du classement alphabétique.

Au moment de formuler une demande, sous forme de "phrase difficile' à traduire", le champ sémantique est délimité implicitement par le fait que la machine ne cherche que des phrases réponses aussi proches que possible de la demande. Le traducteur reçoit un glossaire qui ne se rapporte qu'à son texte et dans l'ordre d'apparition des termes. 
Une mise à jour régulière est prévue qui supprime le problème d'une certaine complétude au départ, et permet d'enregistrer rapidement ces néologismes qui font défaut dans les dictionnaires ordinaires, souvent dépassés dès leur sortie de presse ! Les pertes de temps considérables, occasionnées par des recherches fastidieuses et souvent vaines, seront réduites au strict minimum.

I'établissement d'une véritable collaboration entre les compilateurs du dictionnaire et les utilisateurs mettra à la disposition de tous les donnees dont disposent quelques-uns et qui risquent, autrement d'etre enfouis à tout jamais dans quelque obscur fichier personnel.

Cet instrument souple, puisqu'adaptable à toutes sortes de demandes, peut non seulement faciliter considerablement le travail de son utilisateur, mais lui offre aussi, sans effort supplémentaire de sa part, la possibilité d'un travail plus rapide et de meilleure qualité, ainsi qu'une spécialisation sans peine.

Système maniable, facile à étendre et à corriger, l'outil de travail tel que nous le proposons offre de multiples avantages, et de loin de favoriser l'élimination du traducteur humain, il pourra bien au contraire constituer une aide appréciable dans son travail. 


\section{Notes bibliographiques.}

I.A. MELCUK . Analyse morphologique en traduction automatique. "Problèmes de Cybermétique" Vol. 6 - 1961, pp.207-276.

L.E. DOSTERT, R.R. MACDONALD - Georgetown University Machine Translation Research project . General report 1952 - 1963 (n³0) june 1963.

G. LAURENT , A. BERNARD-GEORGES , D. LEVENBACH - Etude Morphologique du substantif allemand -Grenoble, avril 1962 - EUR 525f. 1964.

J. BLOIS, Fr. DECRESY, J. MOMMENS - Analyse morphologique automatique du français : Université libre de Bruxelles. Mars 1963.

DICAUTOM - La " Traduction Automatique " - 1963 , Vol.IV n³ (Mouton)

L. HIRSCHBERG - Dictionnaires automatiques pour traducteurs humains. " Journal des Traducteurs ", Montréal , Vol.10, n³ (1965)pp. 78-86.

J.A. BACHRACH - Une méthode en terminologie - Comptes rendus du "Colloque de Linguistique Appliquée " -Ceca Luxembourg- avril 1966.

L. HIRSCHBERG - Conception, construction et utilisation de dictionnaires automatiques multilingues. Comptes rendus du " Colloque de Linguistique Appliquée " - Ceca, Luxembourg - avril 1966. 